\title{
ON THE IDEALS AND AUTOMORPHISMS OF NON-ASSOCIATIVE RINGS
}

\author{
M. F. SMILEY
}

Introduction. Relations between the multiplication ring of a ring (=non-associative ring) ${ }^{1}$ and the ring itself have been pointed out by a number of writers [1-7]. In the present note it is shown that the ideal lattice of a ring with unit element is isomorphic to the sublattice of all right ideals of the multiplication ring which contain the annihilator of the unit. A corresponding result is obtained for the right ideal lattice. These results generalize some conditions of R. D. Schafer [7] which describe the simplicity or right simplicity of an algebra with unit element in terms of the right ideal structure of the multiplication ring. We do not quite assume the existence of a unit. (See the paragraph preceding Lemma 2.) By adjoining a unit element, we are able to derive similar, but not quite so precise, results for arbitrary rings.

We also give considerably simplified proofs of the results ${ }^{2}$ of Schafer [7] which concern the automorphisms of rings with unit. Here our simplification consists in avoiding the so-called "reconstruction" of a ring with unit from its multiplication ring. Again we do not quite require a unit.

1. Ideals in rings. Consider a ring $A$. Let $M_{r}\left(M_{l}\right)$ denote the set of all right (left) multiplications $R_{a}: x R_{a}=x a\left(L_{a}: x L_{a}=a x\right)$ of $A$. Let $M_{r}^{*} \equiv[\rho](M \equiv[\mu])$ denote the ring generated by $M_{r}$ (by $\left.M_{r} \cup M_{l}\right)$. Let $N_{r} \equiv\left[\nu_{r}\right](N \equiv[\nu])$ be the right ideal of $M_{r}^{*}$ (of $M$ ) which is generated by the set of elements of $M_{r}^{*}$ of the form $\rho=R_{x y}$ $-R_{x} R_{y}$ (of $M$ of the forms $\mu=R_{x y}-R_{x} R_{y}, \mu=L_{x y}-L_{y} L_{x}$, and $\mu$ $=R_{x}-L_{x}$ ), where $x$ and $y$ are arbitrary elements of $A$.

Lemma 1. If $a \in A, \rho \in M_{r}^{*}$, and $\mu \in M$, then (1) $R_{a} \rho-R_{a p} \in N_{r}$ and (2) $R_{a \mu}-R_{a \mu} \in N$.

Proof. The relations (1) and (2) are additive in $\rho$ and in $\mu$ and we

Received by the editors November 23, 1949.

${ }^{1}$ Following a suggestion of an editor and a referee, we shall use hereaf ter in this note the terms ring and algebra in place of non-associative ring and non-associative algebra. The term naring of [8] will not be used here. Numbers in brackets refer to the references at the end of the paper.

2 Schafer stated these results for algebras of finite order over a base field, but his arguments do not essentially use finite dimensionality except on p. 582, line 13, and in the proof of his Theorem 6. 
may assume that $\rho$ and $\mu$ are products of finitely many elements of $M_{r}$ and of $M_{r} \cup M_{l}$, respectively. We then prove (1) and (2) by induction on the number of factors of $\rho$ and of $\mu$. If $\rho=R_{x}$, then (1) holds by the definition of $N_{r}$. To complete the induction, write $\rho$ $=W R_{y}, R_{a} \rho-R_{a \rho}=R_{a} W R_{y}-R_{a W R_{y}}=R_{a W} R_{y}-R_{(a W) y}+\left(R_{a} W-R_{a W}\right) R_{y}$ $\in N_{r}$. If $\mu=L_{x}$, we obtain (2) by the following computation: $R_{a} L_{x}$ $-R_{a L_{x}}=L_{a} L_{x}-L_{x a}+\left(R_{a}-L_{a}\right) L_{x}-\left(R_{a L_{x}}-L_{a L_{x}}\right) \in N$. To complete the induction, it suffices to note that if $\mu=W L_{y}$, then $R_{a} \mu-R_{a \mu}=R_{a} W L_{y}$ $-R_{a W L_{y}}=R_{a W} L_{y}-L_{y(a W)}+\left(R_{a} W-R_{a W}\right) L_{y}=L_{a W} L_{y}-L_{y(a W)}+\left(R_{a W}\right.$ $\left.-L_{a W}\right) L_{y}+\left(R_{a} W-R_{a W}\right) L_{y} \in N$.

REMARK. We now see that if $A$ has a unit element 1 , then $N_{r}(N)$ consists of all $\rho(\mu)$ such that $1 \rho=0(1 \mu=0)$. For clearly $1 \rho=0$ $(1 \mu=0)$ if $\rho \in N_{r}(\mu \in N)$. Conversely, Lemma 1 with $a=1$ shows that $\rho \in N_{r}(\mu \in N)$ if $1 \rho=0(1 \mu=0)$. Even if $A$ has no unit element, it is easy to see that $c \in A$ is in the center of $A$ if and only if $c \nu=0$ for every $\nu \in N$ (cf. [7, Theorem 8]).

We shall say that $A$ satisfies condition $\mathrm{U}_{l}(\mathrm{U})$ in case $R_{x}=0$ implies that $x=0$ and $M_{r} \cap N_{r}=0\left(M_{r} \cap N=0\right)$. We shall see that the condition $U_{l}(U)$ will replace the assumption of the existence of a left unit element (unit element) in our discussion. That the condition $\mathrm{U}\left(\mathrm{U}_{l}\right)$ is actually weaker than the outright assumption of a unit element (left unit element) may be seen by considering a Boolean ring which has no unit.

REMARK. Nakayama [5] has also indicated a weakening of the requirement that $A$ have a unit. He requires merely that every finite subset of $A$ has a left unit in $A$ and also a right unit in $A$. This condition implies our condition $\mathrm{U}_{l}$, as is easily seen, but to obtain the condition $\mathrm{U}$ we seem to require that each finite subset of $A$ has a unit in $A$.

LEMMA 2. If $\rho \in M_{r}^{*}(\mu \in M)$, then there are elements $a \in A, \nu_{r} \in N_{r}$ $(a \in A, \nu \in N)$ such that $\rho=R_{a}+\nu_{r}\left(\mu=R_{a}+\nu\right)$. These elements are unique if $A$ satisfies the conditon $\mathrm{U}_{l}(\mathrm{U})$.

Proof. Again additivity permits us to assume that $\rho(\mu)$ is a product of finitely many elements of $M_{r}$ (of $M_{r} \cup M_{l}$ ). But then $\rho=R_{a}$ or $\rho=R_{a} \rho_{1}$. In the latter case we have $\rho=R_{a \rho_{1}}+\nu_{r}$ by Lemma 1 . To complete the proof, we must consider $\mu=L_{a}=R_{a}+\left(L_{a}-R_{a}\right)=R_{a}+\nu$, and $\mu=L_{a} \mu_{1}=R_{a} \mu_{1}+\left(L_{a}-R_{a}\right) \mu_{1}=R_{a \mu_{1}}+\nu^{\prime}$, by Lemma 1 . The uniqueness under the stated conditions is obvious.

THEOREM 1. Let $A$ be a ring which satisfies condition U. Then the mapping $I \rightarrow I^{*} \equiv\left[R_{a}+\nu ; a \in I, \nu \in N\right]$ is a lattice isomorphism be- 
tween the ideal lattice of $A$ and the lattice of all right ideals of $M$ which contain $N$.

Proof. Clearly $I^{*}$ contains $N$. To see that $I^{*}$ is a right ideal of $M$, note first that if $a, b \in I, \nu, \nu^{\prime} \in N$, then $\left(R_{a}+\nu\right)-\left(R_{b}+\nu^{\prime}\right)=R_{a-b}$ $+\left(\nu-\nu^{\prime}\right) \in I^{*}$. If $\mu \in M$, we use Lemmas 1 and 2 to compute $\left(R_{a}+\nu\right) \mu$ $=\left(R_{a}+\nu\right)\left(R_{x}+\nu_{1}\right)=R_{a x}+R_{a} R_{x}-R_{a x}+R_{a \nu_{1}}+R_{a} \nu_{1}-R_{a \nu_{1}}+\nu R_{x}+\nu \nu_{1}$ $=R_{a x+a \nu_{1}}+\nu_{2} \in I^{*}$. Thus $I^{*}$ is a right ideal of $M$. Now let $J$ be an arbitrary right ideal of $M$ which contains $N$. Define $I \equiv\left[a ; R_{a} \in J\right]$. Then we verify that $I$ is an ideal of $A$ as follows. Let $a, b \in I, x \in A$. Then $R_{a-b}=R_{a}-R_{b} \in J, R_{a x}=R_{a} R_{x}+R_{a x}-R_{a} R_{x} \in \mathcal{J}$, since $J$ contains $N$, and $R_{x a}=L_{x a}+R_{x a}-L_{x a}=R_{a} L_{x}+\left(L_{a}-R_{a}\right) L_{x}+L_{x a}-L_{a} L_{x}+R_{x a}$ $-L_{x a} \in \mathfrak{J}$, since $\mathfrak{J}$ contains $N$. Thus $I$ is an ideal of $A$. It is easy to see that $I^{*} \leqq J$ and Lemma 2 assures us that $J \leqq I^{*}$. Thus $I^{*}=\mathfrak{J}$, and our mapping is exhaustive. To see that it is one-to-one, let $I^{*}=J^{*}$ for ideals $I$ and $J$ of $A$. If $a \in I$, then $R_{a} \in I^{*}=J^{*}, R_{a}=R_{b}+\nu$ with $b \in J$ and $\nu \in N$. Then $R_{a-b} \in N \cap M_{r}$, and the condition $\mathrm{U}$ yields $a=b \in J$. We have shown that $I \leqq J$, and an interchange of $I$ and $J$ yields $I=J$. To complete the proof, it suffices to remark that if $I \geqq J$, then $I^{*} \geqq J^{*}$ and that $I=\left[a ; R_{a} \in I^{*}\right]$ shows that $I^{*} \geqq J^{*}$ implies that $I \geqq J$. Thus our mapping $I \rightarrow I^{*}$ is one-to-one and preserves order, and is, therefore, a lattice isomorphism.

REMARK. The anti-isomorph of a simple example previously given by us [8] shows that Theorem 1 need not be true when the condition $\mathrm{U}$ is suppressed. This example is an algebra $A$ (over a field $F$ ) with basal units $e$ and $u$ and multiplication table: $e e=u u=e, e u=0$, $u e=u$. It was shown in [8] that $A$ is simple. With respect to the given basis, the right multiplications of $A$ are represented by matrices of the form

$$
R_{x}=\left[\begin{array}{ll}
\alpha & 0 \\
\beta & \alpha
\end{array}\right] \quad(x=\alpha e+\beta u ; \alpha, \beta \in F) .
$$

Thus $M_{r}=M_{r}^{*}$ in our example. If $x^{\prime}=\alpha^{\prime} e+\beta^{\prime} u$, with $\alpha^{\prime}, \beta^{\prime} \in F$, then $R_{x x^{\prime}}-R_{x} R_{x^{\prime}}=\beta^{\prime} R_{y}$, where $y=\beta e-\alpha u$. Thus $N_{r}=M_{r}=M_{r}^{*}$. Since we always have $N \geqq N_{r}$, it is clear that $A$ satisfies neither condition $\mathrm{U}$ nor condition $\mathrm{U}_{l}$. Since

$$
L_{x}=\left[\begin{array}{ll}
\alpha & \beta \\
\beta & 0
\end{array}\right]
$$

we easily see that $M \cong F_{2}$, a total matric ring over $F$. Then we see that $N=M$, by the known form of right ideals of $F_{2}$. The conclusion of Theorem 1 does not hold for $A$. Our example shows also that our 
next theorem is false when the condition $\mathrm{U}_{l}$ is suppressed.

TheOREM 2. Let $A$ be a ring which satisfies the condition $\mathrm{U}_{l}$. Then the mapping $I \rightarrow I^{+} \equiv\left[R_{a}+\nu_{r} ; a \in I, \nu_{r} \in N_{r}\right]$ is a lattice isomorphism between the right ideal lattice of $A$ and the lattice of all right ideals of $M_{r}^{*}$ which contains $N_{r}$.

We shall not give the proof, since it is almost a verbatim repetition of the proof of Theorem 1 .

It is well known that we may embed an arbitrary ring $A$ into a ring $A^{\prime}$ which has a unit element and is such that $A$ is an ideal of $A^{\prime}$. This may be done, of course, in infinitely many different ways. It should be observed that the usual construction also yields the fact that every (right) ideal of $A$ is a (right) ideal of $A^{\prime}$. Then we may obtain a lattice isomorphism of the (right) ideal lattice of $A$ and the sublattice of all right ideals of $M^{\prime}$ (of $\left(M_{r}^{\prime}\right)^{*}$ ) which contain $N^{\prime}\left(N_{r}^{\prime}\right)$ and which are contained in the right ideal $\left[R_{a}+\nu^{\prime} ; a \in A, \nu^{\prime} \in N^{\prime}\right]$ of $M^{\prime}$ (the right ideal $\left[R_{a}+\nu_{r}^{\prime} ; a \in A, \nu_{r}^{\prime} \in N_{r}^{\prime}\right]$ of $\left(M_{r}^{\prime}\right)^{*}$ ).

2. Automorphisms of rings. Let $A$ be a ring which satisfies condition $\mathrm{U}_{l}$ and let $S^{*}$ be an automorphism of $M_{r}^{*}$ such that $N_{r} S^{*}=N_{r}$ and $M_{r} S^{*}=M_{r}$. Then, if $x \in A$, we may define $x S$ uniquely by means of the equation $R_{x} S^{*}=R_{x s}$. We shall prove that the mapping $S$ : $x \rightarrow x S$ is an automorphism of $A$. Clearly $A S=A$, since $M_{r} S^{*}=M_{r}$. Also $x S=y S$ gives $R_{x} S^{*}=R_{y} S^{*}, R_{x}=R_{y}, x=y$ by condition $\mathrm{U}_{l}$. Thus $S$ is a one-to-one mapping of $A$ onto $A$. To see that $S$ is an automorphism of $A$, first compute $R_{x S+y S}=R_{x S}+R_{y S}=R_{x} S^{*}+R_{y} S^{*}$ $=\left(R_{x}+R_{y}\right) S^{*}=\left(R_{x+y}\right) S^{*}=R_{(x+y) S}$. Then condition $\mathrm{U}_{l}$ gives $(x+y) S$ $=x S+y S$. Observe that $\left(R_{x y}-R_{x} R_{y}\right) S^{*}=R_{x y} S^{*}-\left(R_{x} S^{*}\right)\left(R_{y} S^{*}\right)$ $=R_{(x y) S}-R_{x S} R_{y S} \in N_{r}$, since $N_{r} S^{*}=N_{r}$. Now we see that $R_{(x y) S}$ $-R_{(x S)(y S)} \in N_{r}$. Finally, condition $\mathrm{U}_{l}$ gives $(x y) S=(x S)(y S)$, and $S$ is an automorphism of $A$.

If $S_{1}^{*}$ is an automorphism of $M$ which maps each of the sets $N$, $M_{r}$, and $M_{l}$ onto itself, then $N_{r} S_{1}^{*}=N_{r}$, provided that the condition $\mathrm{U}$ is valid. To see this, note that $\left(R_{x y}-R_{x} R_{y}\right) S_{1}^{*}=R_{x y} S_{1}^{*}-\left(R_{x} S_{1}^{*}\right)$ - $\left(R_{y} S_{1}^{*}\right)=R_{u}-R_{v} R_{w}=R_{u}-R_{v w}+\nu_{r}$, where $u, v, w \in A$ and $\nu_{r} \in N_{r}$. Condition $\mathrm{U}$ yields $u-v w=0,\left(R_{x y}-R_{x} R_{y}\right) S_{1}^{*} \in N_{r}$, and it follows that $N_{r} S^{*} \leqq N_{r}$. Since $\left(S_{1}^{*}\right)^{-1}$ is also an automorphism of $M$ satisfying our requirements, we find also that $N_{r}\left(S_{1}^{*}\right)^{-1} \leqq N_{r}$, so that $N_{r} S_{1}^{*}=N_{r}$, as desired.

TheOREM 3. Let $G$ be the group of automorphisms $S$ of a ring $A$ which satisfies condition U. Let $H$ be the group of automorphisms of $M$ which map each of the sets $N, M_{r}$, and $M_{l}$ onto itself. Then the mapping 
$S^{*}: \mu \rightarrow \mu S^{*}=S^{-1} \mu S$ is in $H$ for every $S \in G$, and the mapping $S \rightarrow S^{*}$ is an isomorphism of $G$ onto $H$.

Proof. It is clear that $S^{*}$ is an automorphism of $M$ and that $\left(S^{*}\right)^{-1}=\left(S^{-1}\right)^{*}$. To prove that $S^{*}$ maps $M_{r}$ onto itself, note that $R_{a} S^{*}=S^{-1} R_{a} S=R_{a S} \in M_{r}$, so that $M_{r} S^{*} \leqq M_{r}$, and $M_{r}\left(S^{*}\right)^{-1} \leqq M_{r}$, $M_{r} S^{*}=M_{r}$. The proof that $M_{l} S^{*}=M_{l}$ is dual. The generators of $N$ are carried by $S^{*}$ into $N$, so that $N S^{*} \leqq N, N\left(S^{*}\right)^{-1} \leqq N, N S^{*}=N$. We have proved that $S^{*} \in H$.

Now let $\Sigma \in H$. Then our remarks preceding the statement of Theorem 3 show that $R_{x} \Sigma=R_{x S}$ defines an automorphism $x \rightarrow x S$ of $A$. Then we have $R_{x S}=S^{-1} R_{x} S=R_{x} S^{*}=R_{x} \Sigma$ for every $x \in A$. Dually, we find that $L_{x} \Sigma=L_{x T}$ defines an automorphism $x \rightarrow x T$ of $A$, and $L_{x T}=T^{-1} L_{x} T=L_{x} T^{*}=L_{x} \Sigma$. Then $S=T$, since $R_{x}-L_{x}$ $\in N,\left(R_{x}-L_{x}\right) \Sigma \in N,\left(R_{x S}-L_{x T}\right) \in N, R_{x S}-R_{x T} \in N, x S=x T$ for every $x \in A$ by condition U. Hence $L_{x} \Sigma=L_{x} S^{*}$, and $\mu \Sigma=\mu S^{*}$ for every $\mu \in M$ follows readily since $\Sigma$ is an automorphism of $M$. We have proved that the mapping $S \rightarrow S^{*}$ is onto $H$. That this mapping is a homomorphism is a trivial consequence of its definition. The kernel consists of those elements $S \in G$ such that $S^{*}=I, R_{x}=R_{x} S^{*}=S^{-1} R_{x} S$ $=R_{x S}, x=x S$ for every $x \in A$ by condition $\mathrm{U}, S=I$. Thus the maping $S \rightarrow S^{*}$ is actually an isomorphism and the proof is complete.

The following theorem is proved in similar fashion.

TheOREm 4. Let $G$ be the group of automorphisms $S$ of a ring $A$ which satisfies condition $\mathrm{U}_{l}$. Let $H$ be the group of automorphisms of $M_{r}^{*}$ which map each of the sets $N_{r}$ and $M_{r}$ onto itself. Then the mapping $S^{*}$ : $\rho \rightarrow \rho S^{*}=S^{-1} \rho S$ is in $H$ for every $S \in G$, and the mapping $S \rightarrow S^{*}$ is an isomorphism of $G$ onto $H$.

We conclude with the following theorem on inner automorphisms of $M$.

TheOREM 5. Let $A$ be a ring which satisfies condition $U$ and let $S$ be an automorphism of $A$. Then there exists a nonsingular $\mu_{0} \in M$ such that $S^{-1} \mu S=\mu_{0}^{-1} \mu \mu_{0}$ for every $\mu \in M$ (if and) only if $S \in M$.

Proof. Let the requirement of the theorem hold. Set $\mu=R_{x}$, and use $S^{-1} R_{x} S=R_{x S}, \mu_{0}^{-1}=R_{a}+\nu$ (valid by Lemma 2), to obtain $R_{x S}$ $=\left(R_{a}+\nu\right) R_{x} \mu_{0}=R_{a} R_{x} \mu_{0}+\nu_{1}+R_{y}+\nu_{2}$, where $y=a R_{x} \mu_{0}$, and we have used Lemma 1. Now apply condition $\mathrm{U}$ to find that $x S=a R_{x} \mu_{0}$ $=x L_{a} \mu_{0}, S=L_{a} \mu_{0} \in M$, as desired. 


\section{REFERENCES}

1. A. A. Albert, Non-associative algebras. I. Fundamental concepts and isotopy, Ann. of Math. (2) vol. 43 (1942) pp. 685-708.

2. - The radical of a non-associative algebra, Bull. Amer. Math. Soc. vol. 48 (1942) pp. 891-897.

3. N. Jacobson, $A$ note on non-associative algebras, Duke Math. J. vol. 3 (1937) pp. 544-548.

4. - Structure theory of simple rings without finiteness assumptions, Trans. Amer. Math. Soc. vol. 57 (1945) pp. 228-245.

5. T. Nakayama, Über einfache distributive Systeme unendlicher Ränge, Proc. Imp. Acad. Tokyo vol. 20 (1944) pp. 61-66.

6. T. Nakayama and G. Azumaya, Über einfache distributive Systeme unendlicher Ränge. II, Proc. Imp. Acad. Tokyo vol. 20 (1944) pp. 348-352.

7. R. D. Schafer, Concerning automorphisms of non-associative algebras, Bull. Amer. Math. Soc. vol. 53 (1947) pp. 573-583.

8. M. F. Smiley, An application of a radical of Brown and McCoy to non-associative rings, Amer. J. Math. vol. 72 (1950) pp. 93-100.

The State University of Iowa 